\title{
Estudo epidemiológico e de concordância diagnóstica entre a citologia, colposcopia e histopatologia, em pacientes com diagnóstico de Papilomavírus Humano.
}

\author{
Epidemiological study and diagnostic compatibility \\ among cytology, colposcopy and histopathology in \\ patients with Human Papillomavirus diagnosis.
}

Recebido em: 19/01/2017

Aceito em: $\quad 10 / 04 / 2017$
Heloisa Cristiny de LIMA ${ }^{1}$; Osanir Oliveira da SILVA ${ }^{2}$; Daliana Caldas Pessoa da SILVA ${ }^{1}$; Lenilton Silva da SILVEIRA-JÚNIOR ${ }^{1-3}$ ${ }^{1}$ Laboratório de Imunologia Clínica, Departamento de Análises Clínicas e Toxicológicas, Faculdade de Farmácia, Universidade Federal do Rio Grande do Norte. Rua General Gustavo Cordeiro de Farias, $2^{\circ}$ andar, Petrópolis CEP: 59012-570. Nata, RN, Brasil. ${ }^{2}$ Faculdade Natalense de Ensino e Cultura. Av. Coronel Estevan, 1055 (Praça Pedro II), Bairro do Alecrim. CEP 59030-400. Natal, RN, Brasil. ${ }^{3}$ Centro de Capacitação Educacional (CCE). AV. Conselheiro Aguiar, 1729 Boa Viagem. CEP: 51111-011. Recife, PE, Brasil.

E-mail: leniltonjunior@gmail.com

\section{ABSTRACT}

The diagnosis of intraepithelial lesions and carcinoma of the cervix is performed by combining three methods, namely cytology, colposcopy, and histology. The present study represents a retrospective and descriptive cross-sectional epidemiological study of patients' charts with cytological and colposcopic findings compatible with HPV infection, at the Potengi Clinic, in the year 2012 (Natal/RN). We analyzed 111 medical records of patients diagnosed with HPV and the data collected were age, menarche, the onset of sexual life, marital status, sexual partners, contraceptive methods used and results of cytological, colposcopic and histopathological exams. Patients had a mean age of 30 years, most of whom were married (71.2\%), menarche at 12-year-old, and sexual debut at approximately 17 years, with a mean of 9.4 partners. About $38 \%$ of them use oral contraceptives, $37 \%$ do not use any method and only $10 \%$ use condoms. The concordance between cytology and colposcopy and between cytology and histology was very significant $(\mathrm{rs}=0,953$; $\mathrm{p}<0,05$ ). From the analysis of the present study, it can be concluded that the association of cytological, colposcopic and histological methods is more efficient in the diagnosis of lesions caused by viruses such as HPV and carcinoma and should, therefore, be used together.

Keywords: papillomaviridae; epidemiology; papanicolaou test; colposcopy; biopsy

\section{RESUMO}

O diagnóstico das lesões intraepiteliais e do carcinoma do colo uterino é realizado mediante a associação de três métodos, que são a citologia, colposcopia e histologia. O presente estudo representa uma pesquisa epidemiológica transversal retrospectiva e descritiva de prontuários de pacientes com achados citológicos e colposcópicos, compatíveis com a infecção por HPV, atendidas na clínica Potengi, no ano de 2012 (Natal, RN). Foram analisados 111 prontuários de pacientes diagnosticadas com HPV e os dados coletados foram idade, menarca, início da vida sexual, estado civil, parceiros sexuais, métodos contraceptivos utilizados e resultados de exames citológicos, colposcópicos e histopatológicos. As pacientes tinham média de idade 30 anos, sendo a maioria casada (71,2\%), com menarca aos 12 
anos e início da vida sexual com aproximadamente 17 anos, com média de parceiros de 9,4. Aproximadamente $38 \%$ fazia uso de anticoncepcional oral, $37 \%$ não fazia uso de qualquer método e apenas $10 \%$ fazia uso de preservativo. $\mathrm{O}$ percentual de concordância entre citologia e colposcopia e entre a citologia e histologia foi bastante significativo $(\mathrm{rs}=0,953 ; \mathrm{p}<0,05)$. A partir da análise do presente estudo, pode-se concluir que a associação dos métodos citológicos, colposcópicos e histológicos apresenta maior eficiência no diagnóstico de lesões causadas por vírus como o HPV e carcinoma, devendo, portanto, serem usados em conjunto.

Palavras-chave: papillomaviridae; epidemiologia; teste de papanicolaou; colposcopia; biópsia.

\section{INTRODUÇÃO}

A colpocitologia oncológica, também chamada de exame de Papanicolau, previne o câncer de colo de útero e deve ser realizada em todas as mulheres com vida sexualmente ativa, pelo menos uma vez ao ano. O exame está disponível gratuitamente na rede pública de saúde do país e consiste na coleta de material do colo uterino para análise em laboratório, sendo uma metodologia simples e de baixo custo $(1,2)$. O objetivo do mesmo é detectar células anormais ou cancerosas, também podendo identificar condições não cancerosas como infecção ou inflamação. O sucesso deste, deve-se ao fato de o mesmo poder detectar doenças que ocorrem no colo uterino antes do desenvolvimento do câncer, não sendo, desta forma somente uma maneira de diagnosticar a doença, mas servindo principalmente para determinar o risco de uma mulher vir a desenvolver o câncer (3).

Sabe-se que a colpocitologia oncológica, ou seja, o método de Papanicolau, juntamente à colposcopia e histopatologia são usados em conjunto, a fim de reduzir os erros e aumentar a segurança no diagnóstico das lesões cervicais precursoras do câncer $(4,5)$.

A colposcopia é uma técnica baseada na exploração amplificada dos epitélios do colo do útero, vagina e vulva, cujo objetivo fundamental é diagnosticar as lesões invasivas ou precursoras do câncer do colo uterino (6). A indicação principal desta técnica é localizar o ponto mais importante, ou seja, o local de maior transtorno epitelial, definindo os limites do que deve ser extirpado ou localmente destruído, com o auxílio do colposcópio, abrangendo uma extensa área (4).

A histopatologia é realizada com amostras retiradas de uma superfície suspeita da presença de lesão ou malignidade e está baseada em critérios morfológico-arquitetural e celular, sendo este método considerado padrão ouro do diagnóstico morfológico. Para este exame, é utilizada a classificação de Richart: Neoplasia Intraepitelial Cervical (NIC) graus I, II e III, conforme o grau da lesão, carcinoma e adenocarcinoma in situ (7).

O laudo citológico pode sugerir desde processos inflamatórios, infecciosos, lesões precursoras do câncer, classificadas segundo a nomenclatura de Bethesda (2001) em alterações fortemente compatíveis com lesão (ASC), lesão intraepitelial escamosa de baixo (LSIL) e alto grau (HSIL) e até mesmo lesões de alto grau compatíveis com carcinoma (HSIL/CA) (4).

Tanto a citologia, quanto a colposcopia e a histologia são testes imprescindíveis para o correto manejo das lesões endocervicovaginais, sobretudo aquelas induzidas pelo Papilomavírus Humano (HPV) (4).

Os HPV são vírus capazes de infectar a pele e/ou mucosas, existindo mais de 100 tipos e subtipos de vírus, sendo que cerca de 40 podem infectar o trato ano-genital, causando lesão de baixo e alto graus (8). A transmissão do vírus se dá por contato direto da pele ou mucosa infectadas, sendo que a principal forma é pela via sexual, que inclui contato oro-genital, genital-genital ou mesmo manual-genital. Dessa forma, o contágio com o HPV pode ocorrer mesmo na ausência de penetração vaginal ou anal (9).

Habitualmente as infecções pelo HPV se apresentam como lesões microscópicas ou não produzem lesões, o que se chama de infecção latente. Porém, mesmo quando não há presença de lesões não é possível garantir que o vírus não esteja presente, mas apenas que não está produzindo sintoma. As infecções pelo HPV podem se manifestar pela forma clínica ou subclínica (9).

As lesões clínicas apresentam-se como verrugas ou lesões exofíticas, que são tecnicamente denomina- 
das condilomas acuminados e popularmente chamadas "crista de galo", "figueira" ou "cavalo de crista". Nas mulheres podem aparecer no colo do útero, vagina, vulva, região pubiana, perianal, perineal e anal, além da boca e garganta (9).

A infecção por subtipos oncogênicos do HPV é considerada o fator causal primário para o desenvolvimento da neoplasia invasora do colo do útero, sendo os tipos 16 e 18 considerados de alto risco oncogênico, estando presentes em $70 \%$ dos casos de câncer de colo uterino, enquanto os tipos 6 e 11, encontrados em 90\% dos condilomas genitais e papilomas laríngeos são considerados não oncogênicos (9).

Na história natural dos tumores epiteliais malignos, há duas fases bem diferenciadas: intra-epitelial e angiogênica. Na primeira, as células neoplásicas mostram um aumento da sua densidade nuclear, apresentam crescimento lento, linear, pois a taxa de proliferação se equilibra com a taxa de morte celular ou apoptose, podendo persistir assim durante meses ou anos. A fase angiogênica é provocada pela maior expressão dos fatores de crescimento do endotélio vascular, sendo caracterizada por um crescimento celular rápido, exponencial e pela capacidade de invasão e produção de metástases (6).

A diferenciação das duas fases supracitadas pode ser feita por meio da colposcopia, seguindo a classificação colposcópica de Barcelona (2002). Na fase intraepitelial são observadas lesões de cor branca, com imagens de mosaico e/ou pontilhado, caso as alterações epiteliais sejam acompanhadas de papilas vascularizadas do estroma. Se houver envolvimento das criptas glandulares são observados orifícios glandulares com anéis brancos ou gotas brancas. Na fase angiogênica é observada uma vascularização atípica ou irregular, que constitui um sinal colposcópico bem conhecido, característico do aparecimento de uma lesão mais grave (6).

Quando as condições vaginais são favoráveis, isto é, na ausência de fatores mutagênicos, em particular do HPV, que é de grande relevância na atualidade, e sob condições adversas, o processo metaplásico desenvolve-se de maneira atípica, promovendo o aparecimento de achados colposcópicos anormais, representados pelos seguintes aspectos: epitélio branco plano; epitélio branco denso; pontilhado fino; pontilhado grosseiro; mosaico fino; mosaico grosseiro; iodo parcialmente positivo; iodo-negativo e vasos atípicos (10).

O epitélio branco plano e denso é o achado anormal mais frequentemente observado e, sob a visão colposcópica, é possível observar alterações su- tis na superfície do epitélio escamoso, entre as quais se incluem elevações papilomatosas ou micropapilares, que sugerem a infecção pelo HPV. Contudo, são lesões de menor gravidade, com exceção do epitélio denso que tende a indicar lesões de alto grau. $\mathrm{O}$ pontilhado apresenta-se como pontos vermelhos e, no geral, a manifestação colposcópica é de lesões intraepiteliais de baixo grau. O mosaico é visto como um desarranjo epitelial e são representados como campos brancos, onde quanto maiores e mais densos, mais importantes são ao padrão vascular, que pode estar associado com neoplasia intraepitelial (10).

É possivel dizer que o teste é iodo-negativo quando o citoplasma de células atípicas, que são pobres em glicogênio, impregna-se muito pouco com o corante iodado, ou seja, coram-se pouco. Porém, além desse achado, é levado em consideração também a positividade parcial ao iodo, que é muito frequente diante de infecção pelo HPV, justificado pelos variados graus de maturação que pode apresentar o epitélio. Os vasos atípicos são representados por capilares que se apresentam de várias formas como vírgula; espaguete; saca-rolhas e grampos de cabelo, sendo um importante achado das alterações epiteliais de alto grau (10).

Os achados colposcópicos anormais, com relativa frequência, apresentam-se associados e, podem ser observados em qualquer segmento da mucosa vaginal. São de difícil visualização, sobretudo quando se situam em parede vaginal anterior e posterior. A mais frequente das atipias da mucosa vaginal é o epitélio branco micropapilar, característico de infecção pelo HPV (10), portanto, a reconhecida importância deste vírus na gênese dos processos neoplásicos do trato genital inferior fez com que o colposcopista deixasse de observar tão somente o colo uterino, mas passou a fazer uma minuciosa inspeção das paredes vaginais, como também da vulva e das regiões perineais e perianais $(4,9)$.

De acordo com os dados informados, é possivel dizer que a citologia, de forma isolada, é considerada como um método limitado (11); porém a associação entre citologia, colposcopia e histologia no diagnóstico das lesões intraepiteliais e carcinoma é essencial para uma conduta adequada tanto para a prevenção quanto para o tratamento das lesões (12).

Desse modo o presente trabalho visou avaliar a concordância diagnóstica entre a citologia, colposcopia e histopatologia, por meio de um estudo retrospectivo e descritivo de prontuários de pacientes com infecção por HPV. 
Tabela 1. Características epidemiológicas das pacientes diagnosticadas como portadoras de HPV, em Natal, RN, Brasil (2012)

\begin{tabular}{|c|c|c|}
\hline \multicolumn{3}{|l|}{ Variável } \\
\hline Idade anos (m, dp) & 30,3 & 9,55 \\
\hline \multicolumn{3}{|l|}{ Estado civil (n, \%) } \\
\hline Solteira & 30 & 27,0 \\
\hline Divorciada & 2 & 1,8 \\
\hline Casada & 79 & 71,2 \\
\hline Idade da menarca (m, dp) & 12,0 & 1,09 \\
\hline Idade início vida sexual (m, dp) & 17,5 & 5,9 \\
\hline Número de parceiros sexuais (m, dp) & 9,4 & 9,06 \\
\hline Tabagismo (n, \%) & 3 & 2,7 \\
\hline Etilismo (n, \%) & 7 & 6,3 \\
\hline $\mathrm{m}=$ média; $\mathrm{dp}=$ desvio padrão; $\mathrm{n}=\mathrm{qu}$ & $\%=p$ & entagem \\
\hline
\end{tabular}

Foi realizado um estudo epidemiológico transversal retrospectivo e descritivo, o qual foi desenvolvido em uma clínica privada de atendimento ginecológico, localizada na cidade de Natal, RN, Brasil. A população estudo foi composta por 111 pacientes atendidas, no ano de 2012, que tiveram diagnóstico de HPV e realizaram exame citológico, colposcópico e histopatológico.

Os resultados da citologia utilizaram a nomenclatura Bethesda (2001), os colposcópicos seguiram a classificação colposcópica de Barcelona (2002) e os histológicos foram descritos de acordo com a classificação histológica de Richart (1967). As variáveis coletadas foram planilhadas no Microsoft Excel, em seguida foram geradas tabelas e gráficos com as informações identificadas para representação das mesmas e analisadas no software estatístico IBM SPSS 20.
Este estudo contempla os princípios vigentes da resolução do Conselho Nacional de Saúde (CNS/MS) 466/12 que trata da pesquisa envolvendo seres humanos e foi aprovado pelo Comitê de Ética em Pesquisa do Hospital Universitário Onofre Lopes (CEP/HUOL) com parecer de número 1.324.695.

\section{RESULTADOS E DISCUSSÃO}

Em relação aos dados epidemiológicos, todas as pacientes do estudo tinham diagnóstico de HPV, com idade variando de 16 a 53 anos (média de 30,3 anos), sendo a maioria casada $(71,2 \%)$, com idade de menarca aos 12 anos e uma média de idade para início da vida sexual de aproximadamente 17 anos. O número de parceiros, ao longo da vida destas mulheres, segundo informações fornecidas pelas mesmas, variou de 1 a 30 (média de 9,4 ) e de todas as pacientes, apenas 3 relataram ser fumantes e 7 tinham hábitos de etilismo, conforme dados da Tabela 1.

Os métodos contraceptivos mais usados pelas pacientes estão descritos na Tabela 2, onde aproximadamente $38 \%$ faz uso de anticoncepcional oral, $37 \%$ não faz uso de qualquer método e apenas $10 \%$ faz uso de preservativo.

Tabela 2. Métodos contraceptivos usados pelas pacientes diagnosticadas como portadoras de HPV, em Natal, RN, Brasil (2012).

\begin{tabular}{|l|c|r|}
\hline \multicolumn{1}{|c|}{ Variável } & $\mathbf{n}$ & \multicolumn{1}{c|}{$\%$} \\
\hline Anticoncepcional oral & 42 & 37,8 \\
\hline Condom & 11 & 9,9 \\
\hline Dispositivo intrauterino & 1 & 0,9 \\
\hline Laqueadura & 16 & 14,4 \\
\hline Nenhum método & 41 & 36,9 \\
\hline
\end{tabular}


$100,0 \%$

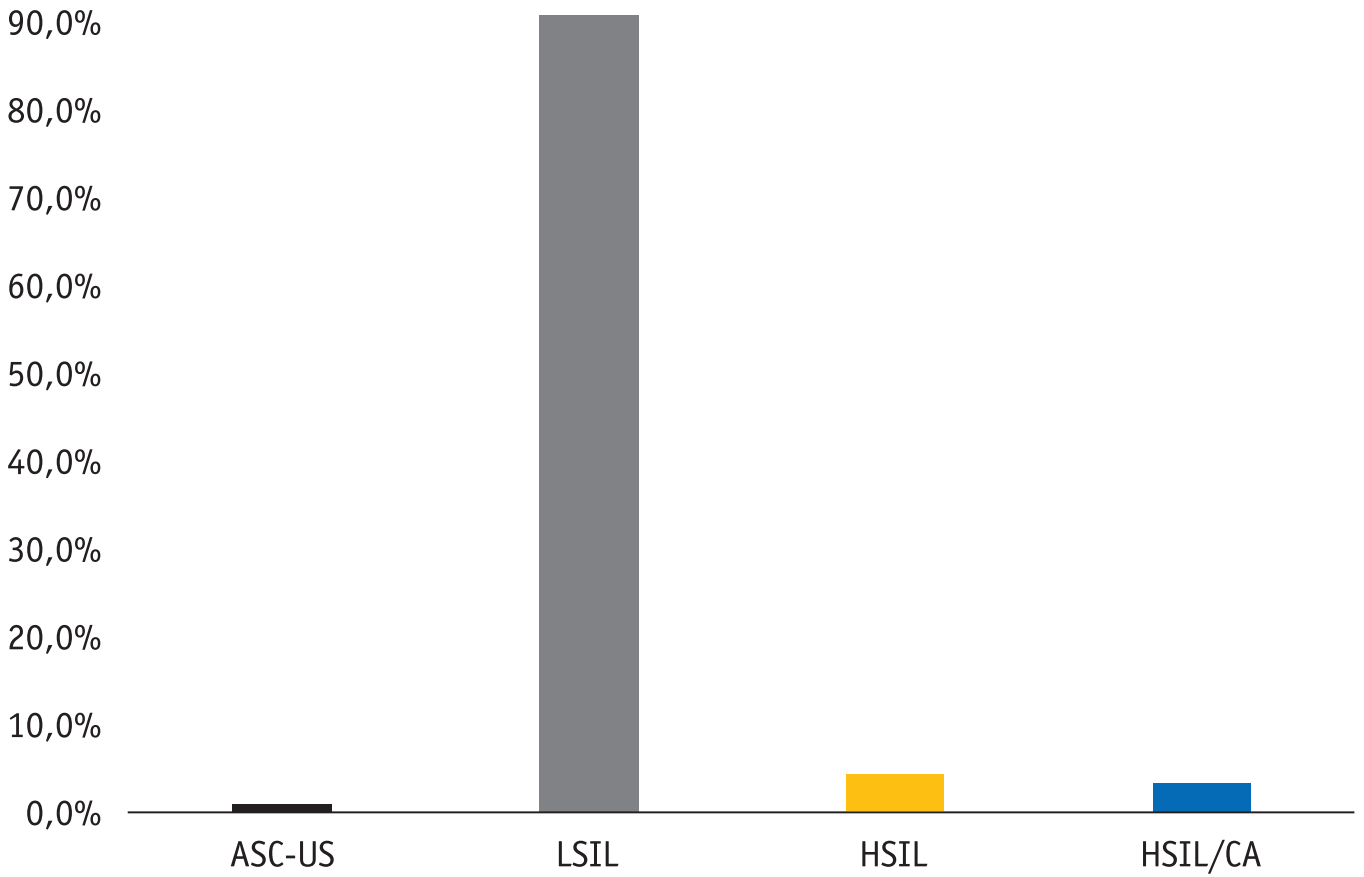

Figura 1: Resultados dos exames de citologia oncótica das pacientes diagnosticadas como portadoras de HPV, em Natal, RN, Brasil (2012). ASC-US (Atipia em células escamosas de significado indeterminado), LSIL (Lesão intraepitelial escamosa de baixo grau), HSIL (Lesão intraepitelial escamosa de alto grau), HSIL/CA (Lesão intraepitelial escamosa de alto grau, não podendo excluir carcinoma).

Foi estabelecida a correlação cito-colpo-histológica para os exames das 111 mulheres que apresentaram resultados citológicos condizentes com lesões, onde foi obtido 1 caso $(0,9 \%)$ de células escamosas atípicas de significado indeterminado (ASC-US), 101 casos (91\%) de lesões intraepiteliais escamosas de baixo grau (LSIL), 5 casos $(4,5 \%)$ de lesões intraepiteliais escamosas de alto grau (HSIL), 4 casos $(3,6 \%)$ de lesões intraepiteliais escamosas de alto grau não excluindo carcinoma epidermoide (HSIL/CA), conforme observado na Figura 1.

Em relação a faixa etária e resultados de citologia oncótica pode ser estabelecido que a idade variou entre 16 e 53 anos, havendo uma incidência maior de ASC-US entre 31 e 45 anos, LSIL entre 16 e 53 anos, HSIL entre 31 e 53 anos e carcinoma epidermóide entre 25 e 42 anos. Em relação aos resultados dos exames colposcópicos (Figura 2), pode ser observado que as alterações menores Zona iodo-negativa $(\mathrm{n}=92)$, pontilhado fino $(n=25)$, mosaico fino $(n=43)$ e epitélio acetobranco $(n=104)$ foram prevalentes quando comparadas às alterações maiores. Sendo assim houve forte concordância entre os achados citológicos (lesões de baixo grau) e colposcópicos (alterações menores). 


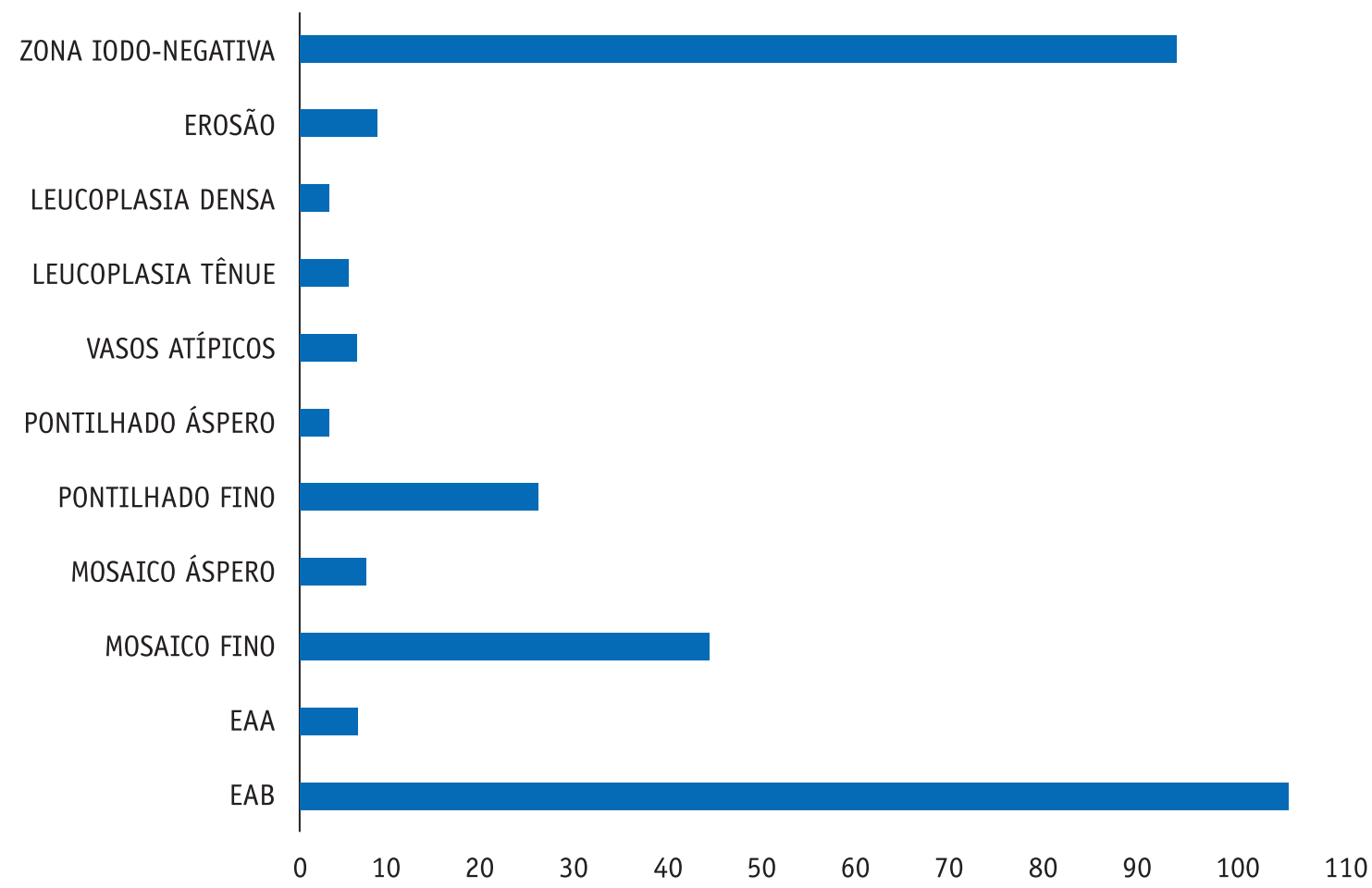

Figura 2. Achados nos exames colposcópicos das pacientes diagnosticadas como portadoras de HPV, em Natal, RN, Brasil (2012). EAA = epitélio acetobranco acentuado; $E A B=$ epitélio acetobranco

A correlação da citologia com a histologia $(\mathrm{rs}=0,953 ; \mathrm{p}<0,05)$ pode ser vista na Figura 3 , onde o único caso de ASC-US e os 101 casos de LSIL foram concordantes com NIC I, os 5 casos de HSIL com NIC II e os 4 casos de HSIL não excluindo carcinoma epidermóide com NIC III.

É esperado que mulheres solteiras, pela possibilidade de um maior número de parceiros, estejam mais vulneráveis a infecção pelo HPV; porém alguns estudos corroboram com nossos achados apontando que a maioria das mulheres com citologia sugestiva de lesão são casadas (13-14). O presente trabalho mostrou que $71 \%$ das mulheres com alguma lesão causada pelo HPV eram casadas, indicando que estas possam estar sob uma maior exposição às doenças infecciosas do trato genital, transmitidas por relação sexual, por muitas vezes devido à confiança na fidelidade de seus companheiros, fazendo com que as mesmas acabem não utilizando métodos de prevenção adequados (13-14).
Além do estado civil das mulheres, um dado epidemiológico importante foi a idade de menarca e do início da vida sexual, pois a menarca pode simbolizar para a menina que a mesma está preparada para o intercurso sexual. O contágio pelo HPV ocorre no início da vida sexual ou por volta dos 20 anos de idade (15), tendo a idade entre 20 e 30 anos uma maior incidência no desenvolvimento do câncer de colo uterino (16). Esse estudo mostrou que a média de idade para menarca foi de 12 anos e que as mulheres tiveram sua primeira experiência sexual com idade média de 17 anos, corroborando com outros trabalhos $(13,17)$ e reforçando a hipótese de que a associação entre a primeira relação e o risco de câncer de colo uterino está cada vez mais ligado, pelo não amadurecimento total da cérvice uterina. Ainda, a realização de práticas sexuais precoce se constitui em fator de risco para o desenvolvimento de lesões, uma vez que na puberdade a zona de transformação cervical nas mulheres jovens está localizada na ectocérvice ficando assim mais exposta e suscetível a infecção pelo HPV durante o ato sexual $(13,17)$. 


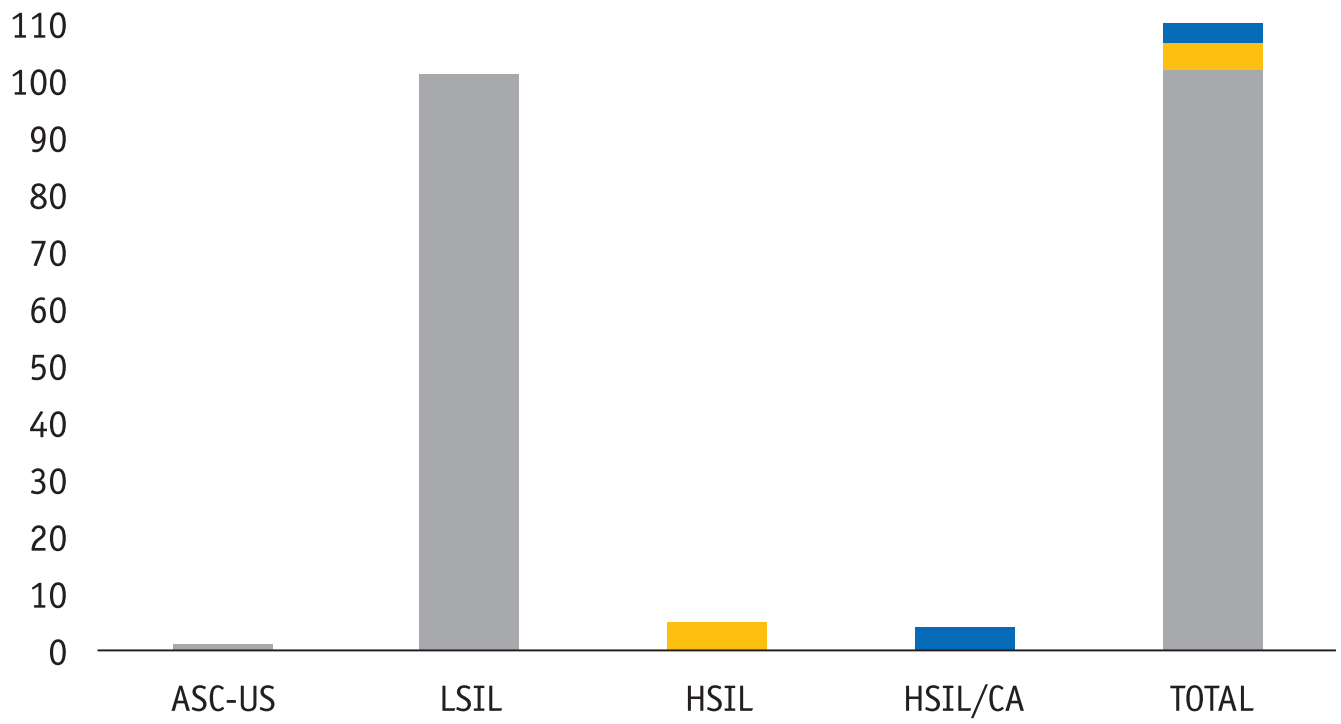

NIC $1 \square$ NIC $2 \square$ NIC 3

Figura 3. Correlação entre os resultados dos exames citológicos e histopatológicos das pacientes diagnosticadas como portadoras de HPV, em Natal, RN, Brasil (2012). ASC-US (Atipia em células escamosas de significado indeterminado), LSIL (Lesão intraepitelial escamosa de baixo grau), HSIL (Lesão intraepitelial escamosa de alto grau), HSIL/CA (Lesão intraepitelial escamosa de alto grau, não podendo excluir carcinoma); NIC 1 (Neoplasia intraepitelial cervical grau 1), NIC 2 (Neoplasia intraepitelial cervical grau 2), NIC 3 (Neoplasia intraepitelial cervical grau 3)

Em relação aos métodos contraceptivos o uso de contraceptivos orais, o uso irregular de preservativo e as baixas condições socioeconômicas, constituem possíveis fatores para o desenvolvimento do câncer de colo de útero (18), que está fortemente relacionado à infecção pelo HPV. Os resultados indicaram que 36,9\% das mulheres não faziam uso de qualquer método contraceptivo, enquanto $63,1 \%$ utilizavam algum método. Dentre eles, o mais usado foi o anticoncepcional oral $(37,8 \%)$, seguido pela laqueadura tubária $(14,4 \%)$, condom $(9,9 \%)$ e dispositivo intrauterino $(0,9 \%)$, conforme a Tabela 2 . Por meio dos dados apresentados, pode ser percebido que do total de mulheres, apenas $9,9 \%$ faziam uso de condom, sendo o único método eficaz para prevenção não somente da gravidez, mas também para infecções sexualmente transmissíveis (IST), como é o caso do HPV, o que ratifica a hipótese de que o fato de ter no matrimônio a segurança de um único parceiro fiel, as mesmas abrem mão de cuidados preventivos para doenças e acabam preocupando-se mais com a contracepção, o que as deixam sob risco de adquirir a infecção pelo HPV e outras IST.

Segundo um estudo multicêntrico realizado pela Internacional Agency for Research on Cancer (IARC) em oito países, incluindo o Brasil, os contraceptivos hormonais orais podem atuar como um importante co-fator no risco de câncer de colo em mulheres com positividade para o HPV cervical. Os resultados mostraram maior risco da doença (cerca de 3 vezes maior), quer para o carcinoma in situ, quer para o invasor, em mulheres HPV positivas e usuárias de pílulas contraceptivas, por cinco ou mais anos, sendo várias as explicações para esses achados. Uma das hipóteses é que os esteroides femininos exógenos atuariam sobre o genoma do vírus, desencadeando estímulos sobre o processo de carcinogênese cervical. Porém, a literatura descarta qualquer possibilidade do contraceptivo facilitar a infecção por este vírus (19).

Ainda com dados do presente estudo, a correlação entre o exame citológico e colposcópico apresentou $93 \%$ de concordância. E já foi demonstrado que a acurácia de achados citológicos e colposcópicos está também na dependência do tamanho da lesão, pois maiores graus de NIC estão relacionados com lesões maiores e mais profundas, facilmente detectadas e graduadas tanto pela citologia quanto pela colposcopia (20).

Os dados da correlação cito-colposcópica também podem ser visualizados na Figura 2, onde pode ser observado que as alterações menores da colposcopia foram prevalentes em relação aos achados maiores, resultados estes que corroboram com os laudos citológicos (Figura 1), uma vez que a presença do HPV está relacionada, no mínimo, a uma LSIL. Logo, o achado de LSIL pode ser correlacio- 
nado com as alterações menores da colposcopia; e os achados de HSIL e HSIL/CA com às alterações maiores.

$\mathrm{Na}$ análise citológica, os laudos de LSIL, HSIL e HSIL/CA devem corresponder, respectivamente, na histologia, a NIC I, NIC II e NIC III. Neste estudo, após análise estatística, os achados citológicos e histológicos, conforme a Figura 3, apresentaram forte correlação $(r s=0,953 ; p<0,05)$ de concordância, evidenciando a importância que a citologia tem como método de triagem e a histologia como método de diagnóstico definitivo.

Outros estudos encontraram valores menores de concordância entre os exames (21-22), porém vale salientar que a própria literatura relata que a concordância entre a citologia e histopatologia possui valor, apenas, quando são colhidas na mesma oportunidade, uma vez que as lesões são passíveis de regressão ou progressão e nos estudos em questão as pacientes foram encaminhadas para a colposcopia, apenas, em um segundo momento quando o resultado da citologia foi sugestivo de atipias celulares.

No presente estudo as coletas se deram na mesma oportunidade, uma vez que no serviço privado, após coleta citológica, a colposcopia é imediatamente realizada e, se necessário, já é feita a coleta do material para a biópsia, o que aumenta a chance de diagnóstico das lesões.

\section{CONCLUSÃO}

Os dados apontaram para a importância da investigação dos fatores associados a infecção pelo HPV, principalmente a idade de menarca e início da atividade sexual. $\mathrm{O}$ estudo mostrou a necessidade de serem introduzidas políticas de orientação aos adolescentes antes de iniciarem a vida sexual para que façam uso de preservativos como principal método preventivo, uma vez que é o único capaz de diminuir e/ou evitar o contágio com o HPV. Quanto ao diagnóstico, a citologia é o melhor método de triagem para a prevenção do câncer de colo uterino e sugere a presença de lesão em sua fase inicial; e que quando bem realizada, o índice de concordância com a colposcopia e histopatologia direciona o médico para diagnóstico e tratamento seguros e eficazes, evitando, assim, o aumento do índice de mortalidade por câncer de colo de útero. É preciso enfatizar que a correlação cito-colpo-histológica é de fundamental importância na identificação das categorias de maior dificuldade diagnóstica.

\section{AGRADECIMENTOS}

À Dra Célia Maria de Menezes Souza, assim como a toda equipe do Laboratório Potengi pela cordialidade e parceria na execução deste trabalho e à Professora Dra Tereza Neuma de Souza Brito pela análise estatística.

\section{REFERÊNCIAS}

1. Dangelo, JG; Fattimi, CA. Anatomia sistêmica e segmentar. 2.ed. São Paulo: Atheneu, 2003.

2. Ormonde Junior, JC; Oliveira, LD; Sá, RM. Fatores de adesão e não adesão das mulheres ao exame colpacitológico. Rev. Gestão \& Saúde. 2015; 6(1):184-200.

3. Brenna, SMF; Hardy, E; Zeferino, LC; Namura, I. Conhecimento, atitude e prática do exame de Papanicolau em mulheres com câncer de colo uterino. Cad. Saúde Pública. 2001; 17(4)909-914. DOI: 10.1590/S0102311X2001000400024

4. Martins, NV. Patologia do trato genital inferior. Capítulo 1. São Paulo: Editora Roca Ltda, 2005.

5. Munhoz, LMBS; Colturato, PL; Borba, TAG; Gonçalves, S; Merlin, JC; Haas, P. Comparativo citológico, colposcópico e histológico de biópsias do colo uterino no ambulatório Amaral Carvalho/Itararé-SP. RBAC. 2009; 41(3):167-171.

6. Moutinho, JM. Colposcopia. [base de dados da internet]. Federação das Sociedades Portuguesas de Obstetrícia e Ginecologia. Capítulo 41. 2014.
7. Stival, CO; Lazzarotto, M; Rodrigues, YB; Vargas, VRA. Avaliação comparativa da citologia positiva, colposcopia e histologia: Destacando a citologia como método de rastreamento do câncer do colo do útero. RBAC. 2005; 37(4):215-218.

8. Nakagawa, JTT; Schirmer, J; Barbieri, M. Vírus HPV e câncerde colo de útero. RevBras Enferm. 2010; 63(2):307311. DOI: 10.1590/S0034-71672010000200021.

9. INCA BMS. HPV e Câncer. [base de dados da internet]. Acesso em 21 de julho de 2015. Disponível em http:// www1.inca.gov.br/ conteudo_view.asp?id=2687.

10. Focchi J; Netto AR. Colposcopia Atual. Porto Alegre: Artmed/Panamericana Editora, 2004.

11. Lapin, GA; Derchain, SFM; Tambascia, J. Comparação entre a colposcopia oncológica de encaminhamento e a da gravidade das lesões cervicais intraepiteliais. Rev. Saúde Pública. 2000; 34(2):120-125. DOI: 10.1590/ S0034-89102000000200004

12. Junior, JE; Cavalcante, JR; Santiago, RO; Silva, DS. Citologia oncótica, colposcopia e histologia no diagnóstico 
de lesões epiteliais do colo uterino. News Lab. Edição 63, 2004.

13. Saiwori, JS; Bezerra, PCG; Franco, ES; Pinheiro, AKB. Perfil de mulheres portadoras de lesões cervicais por HPV quanto aos fatores de risco para câncer de colo uterino. DST - J bras Doenças Sex Transm. 2005; 17(2):143148.

14. Chih, HJ; Lee, AH; Colville, L; Xu, D; Binns, CW. Condom and oral contraceptive use and risk of cervical intraepithelial neoplasia in Australian women. J Gynecol Oncol 2014; 25(3):183-187. DOI: 10.3802/ jgo.2014.25.3.183

15. Cirino, FMSB; Nichiata, LYI; Borges, ALV. Conhecimento, atitude e práticas na prevenção do câncer de colo uterino e HPV em adolescentes. Esc Anna Nery Rev Enferm. 2010; 14(1):126-134. DOI: 10.1590/S141481452010000100019 .

16. Motta, EV; Fonseca, AM; Bagnoli, VR; Ramos, LO; Pinotti, JA. Colpocitologia em ambulatório de Ginecologia Preventiva. Rev Ass Med Brasil. 2001; 47(4):302-310. DOI: $10.1590 / \mathrm{S} 0104-42302001000400032$

17. Silva, EO; Coelho, MCV; Athayde, LA. Alterações citológicas associadas a infecção pelo Papilomavirus Huma- no em mulheres atendidas em um hospital. Rev. Gestão \& Saúde. 2016; 7(1):52-64.

18. Bosh. FX; Sanjosé, S. Human papillomavirus and cervical cancer- burden and assessment of causality. Jornal of National Cancer Institute Monographs. 2003; 31:3-13.

19. Mendes, J; Santos, AP; Alberto, C. Contracepção Hormonal Oral, HPV e risco de Câncer cérvico-uterino. Rev Assoc Med Bras, 2002; 48(2):93-117. DOI: 10.1590/ S0104-42302002000200005

20. Alves, RRF; Teixeira, TS; Netto, JCA. Performance da citologia e colposcopia frente a histologia no rastreamento e diagnóstico das lesões precursoras do colo uterino. DST - J Bras Doenças Sex Transm. 2002; 14(5):33-38

21. Carvalho, ME; Stofler, W; Nunes, RD; Rojas, PFB; Trapani-Junior, A; Schneider, IJC. Avaliação do desempenho da citologia e colposcopia comparados com a histopatologia no rastreamento e diagnóstico das lesões do colo uterino. ACM. 2011; 40(3):30-36.

22. Katz, LMC; Souza, ASR; Fittipaldi, SO; Santos, GM; Amorim, MMR. Concordância entre citologia, colposcopia e histopatologia cervical. Rev Bras Ginecol Obstet. 2010; 32(8):368-373. DOI: 10.1590/S010072032010000800002 